\title{
Perspectives d'applications de la programmation linéaire aux industries laitières
}

\author{
par \\ M. BEN RAJEB et J. J. BIMBENET \\ Chaire de "Génie Industriel Alimentaire " \\ de l'Ecole Nationale Supérieure des Industries Agricoles et Alimentaires \\ C.E.R.D.I.A. - 91305 Massy
}

\section{LA PROGRAMMATION LINEAIRE}

Expliquons sur l'exemple d'un mélange en quoi consiste la programmation linéaire.

Soit une série de produits que l'on recherche à combiner pour obtenir un mélange devant obéir à certaines contraintes. Voici un exemple (imaginaire) de telles contraintes :

Humidité moins de 62 p. 100.

Matière grasse au moins 40 p. 100.

Amidon 10 p. 100 exactement.

Vitamine $\mathrm{X}$ au moins 50 unités.

Cendres entre 2 et 5 p. 100.

Couleur entre 12 et 14 unités.

etc.

Toutej ces contraintes étant posées, on ne pourra résoudre le problème que si l'on connaît les caractéristiques correspondantes de tous les éléments du mélange, que l'on classe selon le tableau ci-dessous. Enfin et surtout, on y porte les prix de chacun de ces composants (tab. 1) :

La programmation linéaire est une méthode mathématique permettant de déterminer le mélange le moins cher satisfaisant aux contraintes que l'on s'est posées.

Cette définition, que nous avons cherché à simplifier et à rendre concrète, doit être éclairée par plusieurs remarques.

Tout d'abord, la programmation linéaire suppose que les critères choisis se combinent linéairement dans le mélange. C'est ainsi que si l'on appelle $\mathrm{x}_{1}, \mathrm{x}_{2}, \mathrm{x}_{3}, \ldots, \mathrm{x}_{2}$ les pourcentages respectifs des produits $1,2,3, \ldots, 20$ dans le mélange $M$, l'humidité $\mathrm{H}_{\mathrm{M}}$ de ce mélange sera en proportion de ces pourcentages, c'est-à-dire que :

$$
100 \mathrm{H}_{\mathrm{s}}=40 \mathrm{x}_{1}+70 \mathrm{x}_{2}+18 \mathrm{x}_{3}+\ldots+25 \mathrm{x}_{2}
$$


TABLEAU 1

\begin{tabular}{|c|c|c|c|c|c|c|c|}
\hline & Humidité & Matière grasse & Amidon & Vitamine $\mathrm{X}$ & Cendres & Couleur & Prix \\
\hline Produit 1 & 40 p. 100 & 10 p. 100 & 10 p. 100 & $80 \mathrm{U}$ & 4 p. 100 & 8 & $25 \mathrm{~F} / \mathrm{kg}$ \\
\hline Produit 2 & 70 & 20 & 3 & 0 & 2 & 16 & 18 \\
\hline Produit 3 & 18 & 55 & 15 & 100 & 7 & 0 & 41 \\
\hline$\ldots \ldots \ldots$ & $\ldots \ldots$ & $\ldots \ldots$ & $\ldots \ldots$ & $\ldots \ldots \ldots$ & $\ldots \ldots \ldots$ & $\ldots \ldots \ldots$ & \\
\hline Produit 20 & ........ & ........ & ......... & $\begin{array}{l}\cdots \cdots \\
60\end{array}$ & ....... & $\ldots \ldots$ & $\cdots$ \\
\hline $\begin{array}{c}\text { Contraintes sur le } \\
\text { mélange }\end{array}$ & $<62$ p. 100 & $\geqslant 40$ p. 100 & $=10$ p. 100 & $>50$ & $2-5$ p. 100 & $12-14$ & minimum \\
\hline
\end{tabular}


Cette loi linéaire est également respectée par tous les autres éléments de la composition du mélange (matière grasse, amidon, vitamines, cendres, etc.).

Toutefois, si l'on veut qu'un critère différent, tel que la couleur, puisse être aussi pris en compte dans le calcul, il est indispensable qu'il obéisse à la même loi : que la couleur du mélange, pour garder cet exemple, soit proportionnelle aux couleurs et aux pourcentages de ses constituants. Ce n'est, cette fois, pas évident, et le problème consistera à définir une échelle objective de mesure de la couleur telle que cette condition soit remplie. Il en sera de même chaque fois que l'on voudra faire intervenir un critère organoleptique (couleur, texture, goût, etc.) dans le calcul.

Il convient, d'autre part, de préciser que la recherche du mélange le moins cher n'est qu'une des applications de la programmation linéaire. D'une façon générale, cette technique s'applique en effet à tout système d'équations ou d'inéquations linéaires assorti d'un critère à maximiser ou minimiser. On peut l'utiliser pour chercher la meilleure affectation de machines d'un atelier, ou un programme optimal de fabrication. La plupart des ouvrages de recherche opérationnelle $[1,2,3,4]$ donnent des exemples de ces applications. On y trouve aussi des explications complètes et rigoureuses sur l'aspect mathématique de la programmation linéaire.

La détermination de la solution peut, dans des cas très simples, être graphique [5]. Une solution numérique manuelle existe pour des cas moins simples. En réalité, lorsque l'on fait appel à cette méthode, c'est toujours pour des cas où le bon sens ne suffit pas, donc pour des cas compliqués. La seule méthode pratiquement utilisable est alors le calcul sur ordinateur.

\section{EXEMPLES D'APPLICATIONS DANS LES INDUSTRIES ALIMENTAIRES}

Nous nous sommes donné pour objectif, dans le cadre d'un projet de recherche en collaboration université-industrie, de détecter dans les industries alimentaires les opérations de mélange susceptibles d'être améliorées par la programmation linéaire, et de montrer, par une recherche sur des exemples précis, la "faisabilité " et l'intérêt de la méthode.

Nous avons classé ces exemples par ordre de difficulté croissante.

\section{a) Optimisation d'un vin de consommation courante}

Le problème qui se posait à nous consistait à minimiser le prix de revient d'un vin ordinaire, à composer à partir d'une palette d'un grand nombre de vins différents ayant chacun un profil analytique donné. Les critères de composition (alcool, acidité, etc.) et certains 
critères̀ organoleptiques simples n'ont posé aucun problème. En revanche, des contraintes liées à la technique de fabrication ont dû être prises en compte d'une façon partiellement empirique.

\section{b) Optimisation d'un produit de charcuterie industrielle}

$\mathrm{Si}$ le choix des matières premières était cette fois plus limité, la difficulté résidait dans la mise au point du système de contraintes technologiques qui nous assuraient que le produit calculé puisse être fabriqué et soit au moins d'une qualité équivalente au produit fabriqué d'après la formule classique. Certains de ces critères technologiques posent des problèmes de mesure objective et de linéarité.

\section{c) Optimisation d'une farine.}

Compte tenu des froments qui existent sur le marché, comment doit-on procéder pour obtenir une farine donnée alors que les renseignements donnés par le "Chopin » ou par le "Brabender " ne sont pas linéaires ? Une étude détaillée des critères envisagés nous a permis de mieux approcher la formulation des farines.

\section{d) Optimisation d'une margarine}

Ce cas d'optimisation est un des plus complexes qui soit. Il est en effet très difficile de définir les contraintes, de les mesurer et surtout de les linéariser. Une approche particulière nous a toutefois permis d'utiliser la programmation linéaire à la résolution de ce problème.

\section{UN EXEMPLE D'APPLICATION EN INDUSTRIE LAITIERE : OPTIMISATION D'UNE CREME GLACEE}

Rappelons qu'une crème glacée, sur le plan de la formulation, est une émulsion à base de lait, de matières grasses, de sucres, et de matières ajoutées.

Ces matières ajoutées, comme le chocolat, le pralin, les œufs, etc., sont indispensables à la crème glacée et grèvent son prix de revient de 10 à 20 p. 100 . Malheureusement elles sont en général spécifiques et donc non interchangeables.

Les matières de base, elles, ne sont pas spécifiques et représentent environ 50 p. 100 du coût de fabrication. D'où l'idée de chercher s'il y a moyen d'optimiser le choix de ces matières premières.

Quelles sont-elles?

- Le lait : L'approvisionnement en lait peut être extrêmement diversifié. En effet, l'industriel a le choix entre le lait entier qu'il peut acheter suivant son taux de matières grasses, un lait écrémé, un lait en poudre écrémé ou non, un lait concentré sucré ou non. 
- Les matières grasses : Ce sont les matières grasses du lait et proviennent de crème fraîche, de beurre, du lait entier ou de la poudre de crème de lait entier.

Les matières grasses végétales peuvent être envisagées, dans la mesure où la législation du pays les autorise, à condition qu'elles aient un goût tout-à-fait neutre et soient faciles à mettre en émulsion.

- Les sucres : Les sucres peuvent être des sucres classiques de betteraves ou de canne (saccharose), des sucres simples du type dextrose ainsi que d'autres produits sucrants qui sont classés dans le commerce d'après leur D.E. ( " dextrose equivalent ») ou leur pouvoir sucrant.

Il faut retenir de ce rapide tour d'horizon des matières premières de base, qu'il est possible de trouver une gamme assez large de chaque produit et qu'un choix doit être fait pour présenter au consommateur le produit de qualité qu'il attend, et ceci au moindre prix.

Pour calculer notre crème glacée, nous avons fixé les différentes spécifications du «mix ». Ces spécifications découlent d'une part de la législation qui détermine la qualité d'une crème glacée d'après son " taux de butyrique » et d'autre part de l'expérience acquise : par exemple, l'industriel sait qu'il doit équilibrer son mix en sucre et en matière sèche dégraissée du lait. Les contraintes organoleptiques entrent donc de façon indirecte dans le calcul.

Pour respecter le secret professionnel de l'entreprise qui a bien voulu nous aider à faire cette démonstration, nous allons appeler les divers ingrédients : $\mathrm{X} 1, \mathrm{X} 2, \ldots, \mathrm{XN}$ et les différentes contraintes : CG1, CG2, ..., CGM.

En pratique, nous nous sommes limités à ne choisir nos différentes matières premières que dans la gamme des produits qui existent en stock à l'usine pour éviter, pour cette démonstration, la critique classique qui consiste à juger suspecte toute matière première inhabituelle.

Malgré cette restriction, et en ayant respecté toutes les contraintes imposées par l'industriel, la solution optimisée s'est révélée moins chère de 3,5 p. 100. Nous l'avons alors fabriquée à petite échelle et fait déguster par le jury de dégustation de l'usine. La formule de l'ordinateur a été unanimement jugée plus équilibrée que la formule traditionnelle.

Pour confirmer la reproductibilité de notre méthode de travail, nous avons refait ce calcul pour 3 produits tout à fait différents quant à leur texture, leur présentation et leurs caractères organoleptiques. Le jury a toujours confirmé la qualité des crèmes glacées optimisées, qui sont pourtant toutes moins chères de 3 à 5 p. 100 que celles de l'industriel. 
La programmation linéaire ne nous donne pas seulement la formule optimale de la crème glacée que nous désirons fabriquer, mais encore le coût marginal des matières premières et le prix de chaque point de contrainte autour de l'optimum, comme le montre le tableau 2 de résultats.

\section{TABLEAU 2}

Optimisation de la formule d'une crème glacée par programmation linéaire : tableau des résultats

Nom de fonction économique : Feco - Valeur : 0.81083 E 03

Numéro de second membre utilisé : 1

\begin{tabular}{|c|c|c|c|}
\hline Activité & Niveau d'activité & Coût original & Coût marginal \\
\hline $\begin{array}{l}\text { CG2 } \\
\text { CG3 } \\
\text { CG4 } \\
\text { CG5 } \\
\text { CG8 } \\
\text { CG9 } \\
\text { X1 } \\
\text { X2 } \\
\text { X3 } \\
\text { X4 } \\
\text { X6 } \\
\text { X8 } \\
\text { X9 } \\
\text { X10 } \\
\text { X11 } \\
\text { X12 }\end{array}$ & 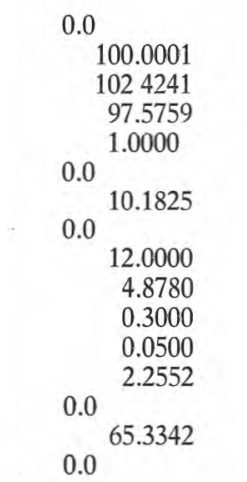 & $\begin{array}{rr}0.0 & \\
0.0 & \\
0.0 & \\
0.0 & \\
0.0 & \\
0.0 & \\
& 40.0000 \\
& 50.0000 \\
& 11.8000 \\
& 9.5000 \\
& 62.4000 \\
& 36.6600 \\
22.7500 \\
23.6500 \\
2.2000 \\
0.0010\end{array}$ & 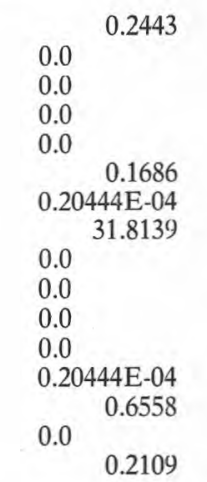 \\
\hline
\end{tabular}

\begin{tabular}{l|r|r|r}
\hline \multirow{2}{*}{ Ligne } & Niveau d'activité & Contrainte & \multirow{2}{*}{ Valeur du dual } \\
\cline { 3 - 4 } & & & \\
\cline { 3 - 4 } CG1 & 1000.0000 & 1000.0000 & -0.4233 \\
CG2 & 8000000 & 800.0000 & -0.2443 \\
CG3 & 799.9998 & 900.0000 & 0.0 \\
CG4 & 3407.4238 & 3305.0000 & 0.0 \\
CG5 & 3407.4238 & 3305.0000 & 0.0 \\
CG6 & 16.0000 & 16.0000 & -12.0099 \\
CG8 & 4.0000 & 3.0000 & 0.0 \\
CG9 & 4.0000 & 4.0000 & 0.1686 \\
CG11 & 0.3000 & 0.3000 & -62.6099 \\
CG13 & 0.0500 & 0.0500 & -36.8699 \\
CG & 95.0000 & 95.0000 & 0.2099 \\
\hline
\end{tabular}

Ainsi, le fait d'utiliser 1 p. 100 de X2 en plus nous coûtera 31,8 de plus alors que toute autre matière première en supplément ne grèvera le prix de revient que de 0,21 au maximum. De même, si 
nous avions exigé 1 p. 100 de plus pour la contrainte CG1, cela nous aurait coûté 42 alors que 1 p. 100 de plus de CG6 ne coûte que 12 , toutes choses étant égales par ailleurs.

Nous voyons tout l'intérêt qu'un industriel soucieux de rester compétitif tout en améliorant ses produits peut tirer d'un tel travail. Rien ne l'empêche par exemple de calculer l'influence d'un rapport sucre/matière grasse sur la qualité organoleptique de son produit et sur le prix de revient.

\section{CONCLUSION}

La formulation de la crème glacée est un exemple, sans doute parmi les plus faciles (si l'on s'en tient à ces simples contraintes sur la composition) des progrès que fera sans doute accomplir la programmation linéaire aux industries laitières.

D'autres viennent à l'esprit, certains peut-être aussi simples, mais les autres sans doute plus difficiles :

- laits gélifiés,

- fromages frais,

- fromages fondus.

Cette liste n'est pas limitative : elle dépend avant tout de l'imagination des spécialistes. Cependant, rappelons-nous que la méthode n'est applicable qu'à des produits de mélange.

Mais il faut surtout souligner que dans la plupart des cas, une applications nouvelle devra faire l'objet d'une recherche avant la phase d'exploitation proprement dite. On doit, en effet, tout d'abord analyser le problème : liste des composants possibles, liste des contraintes de toute sorte, compositions analytiques et organoleptiques éventuelles de tous les composants. La deuxième étape consiste à vérifier la linéarité des contraintes prises en considération, et, le cas échéant, à essayer de les linéariser par l'une des méthodes mathématiques classiques. Lorsque ce n'est pas possible, il faut se rabattre sur une méthode semi-empirique ou même empirique pour tenir compte du phénomène. Enfin des considérations liées aux circonstances particulières dans lesquelles travaille l'usine (approvisionnements, stocks, diverses contraintes de fabrication, de vente, etc.) peuvent et doivent être prises en compte de façon quantitative ou qualitative.

L'ensemble de ces recherches est certainement justifié par les chances d'un abaissement, même limité, du prix de revient ou d'une meilleure maîtrise des éléments de la formulation des produits. Aussi avons-nous la conviction qu'elles seront entreprises et que la programmation linéaire constituera dans peu d'années un outil très répandu au service des industries laitières. 


\section{S u m m a r y}

Linear programming is a classical mathematical technique of optimization, easy to operate on a computer, which may be of a great utility in food formulation, specially for blends of some dairy products. The mixture must be defined by certain constraints, which may be analytical, technological, organoleptic or legislative, provided that all are linear. The computer gives the less costly formula fitting with those constraints. An exemple is given in ice cream blending.

\section{Références bibliographiques}

[1] Kaufmann (A.) et Faure (R.) (1968). - Invitation à la recherche opérationnelle. Dunod, Paris.

[2] Desbazeille (G.) (1964). - Exercices et problèmes de recherche opérationnelle. Dunod, Paris.

[3] Kaufmann (A.) (1964). - Méthodes et modèles de la recherche opérationnelle. Dunod, Paris, (tome 1).

[4] Maurin (H.) (1967). - Programmation linéaire appliquée. Editions Technip, Paris.

[5] Ben Rajeb (M.), Loncin (M.) et Bimbenet (J. J.) (1971). - Optimisation des mélanges alimentaires par la programmation linéaire. Industr. Alim. Agric., $88,1223$. 